\title{
A Numerical Method for Compressible Model of Contamination from Nuclear Waste in Porous Media
}

\author{
Zhifeng Wang \\ School of Mathematics and Information Sciences, Yantai University, Yantai 264005, China \\ Correspondence should be addressed to Zhifeng Wang; wangzhifeng1997@163.com
}

Received 6 September 2020; Accepted 25 April 2021; Published 19 May 2021

Academic Editor: Yong Hong Wu

Copyright (c) 2021 Zhifeng Wang. This is an open access article distributed under the Creative Commons Attribution License, which permits unrestricted use, distribution, and reproduction in any medium, provided the original work is properly cited.

This paper studies and analyzes a model describing the flow of contaminated brines through the porous media under severe thermal conditions caused by the radioactive contaminants. The problem is approximated based on combining the mixed finite element method with the modified method of characteristics. In order to solve the resulting algebraic nonlinear equations efficiently, a two-grid method is presented and discussed in this paper. This approach includes a small nonlinear system on a coarse grid with size $H$ and a linear system on a fine grid with size $h$. It follows from error estimates that asymptotically optimal accuracy can be obtained as long as the mesh sizes satisfy $H=O\left(h^{1 / 3}\right)$.

\section{Introduction}

A compressible nuclear waste disposal contamination problem in porous media is presented by the following coupled systems of partial differential equations. The physical processes can be concreted to be a high-level waste disposal buried in a salt dome, and next the salt dissolves to generate a brine, radioactive elements decay to generate heat, and finally the radionuclides are transported by the flow.

Fluid:

$$
\begin{gathered}
\phi_{1} \frac{\partial p}{\partial t}+\nabla \cdot u=-q+R_{s}^{\prime}(\widehat{c}), \\
u=-\frac{k}{\mu(\widehat{c})} \nabla p=-a(\widehat{c})^{-1} \nabla p,
\end{gathered}
$$

where $p$ and $u$ are the fluid pressure and Darcy velocity, respectively, $\phi_{1}=\phi c_{w}$ and $\phi$ is the porosity. $q=q(x, t)$ is a production term, $R_{s}^{\prime}(\widehat{c})=\left[c_{s} \phi K_{s} f_{s} /\left(1+c_{s}\right)\right](1-\widehat{c})$ is a salt dissolution term, $k(x)$ is the permeability of the rock, and $\mu(\widehat{c})$, the viscosity of the fluid, is dependent upon $\widehat{c}$, the concentration of the brine in the fluid.

Brine:

$$
\phi \frac{\partial \widehat{c}}{\partial t}+u \cdot \nabla \widehat{c}-\nabla \cdot\left(E_{c} \nabla \widehat{c}\right)=g(\widehat{c})
$$

where $E_{c}$ is the diffusion tensor including the molecular diffusion and mechanical diffusion and $E_{c}=\mathbf{D}+D_{m} \mathbf{I}$, $\mathbf{D}=(D)_{i, j}=\left(\left(\alpha_{T}|u| \delta_{i, j}+\left(\alpha_{L}-\alpha_{T}\right) u_{i} u_{j}\right) /|u|\right)$, and $g(\widehat{c})=$ $-\widehat{c}\left\{\left[c_{s} \phi K_{s} f_{s} /\left(1+c_{s}\right)\right](1-\widehat{c})\right\}-q_{c}+R_{s}$. Here, $D_{m}$ is molecular diffusion. $u_{i}$ and $u_{j}$ are two direction cosines of Darcy velocity. $\mathbf{I}$ is an identity matrix.

Heat:

$$
d_{1}(p) \frac{\partial p}{\partial t}+d_{2} \frac{\partial T}{\partial t}+c_{p} u \cdot \nabla T-\nabla \cdot\left(\widetilde{E}_{H} \nabla T\right)=Q(u, T, \widehat{c}, p)
$$

where $T$ is the temperature of the fluid, $d_{1}(p)=-\phi c_{w}\left[U_{0}+(p / \rho)\right], d_{2}=\phi c_{p}+(1-\phi) \rho_{R} c_{p R}, \widetilde{E}_{H}=$ $\mathbf{D} c_{p w}+K_{m}^{\prime} \mathbf{I}, \quad K_{m}^{\prime}=k_{m} / \rho_{0}$, and $Q(u, T, \widehat{c}, p)=-\left\{\left[\nabla U_{0}-\right.\right.$ $\left.\left.c_{p} \nabla T_{0}\right] \cdot u+\left[U_{0}+c_{p}\left(T-T_{0}\right)+(p / \rho)\right]\left[-q+R_{s}^{\prime}\right]\right\}-q_{L}-$ $q H-q_{H}$.

Radionuclide (component $i$ ):

$$
\begin{aligned}
& \phi K_{i} \frac{\partial c_{i}}{\partial t}+u \cdot \nabla c_{i}-\nabla \cdot\left(E_{c} \nabla c_{i}\right)+d_{3}\left(c_{i}\right) \frac{\partial p}{\partial t} \\
& =f_{i}\left(\widehat{c}, c_{1}, \ldots, c_{N}\right), \quad i=1, \ldots, N,
\end{aligned}
$$


where $c_{i}$ is the trace concentration of the $i$ th radionuclide, $d_{3}\left(c_{i}\right)=\phi c_{w} c_{i}\left(K_{i}-1\right)$, and

$$
\begin{aligned}
f_{i}\left(\widehat{c}, c_{1}, \ldots, c_{N}\right)= & c_{i}\left\{q-\left[\frac{c_{s} \phi K_{s} f_{s}}{\left(1+c_{s}\right)}\right](1-\widehat{c})\right\}-q c_{i}-q_{c i} \\
& +q_{0 i}+\sum_{j=1}^{N} k_{i j} \lambda_{j} K_{j} \phi c_{j}-\lambda_{i} K_{i} \phi c_{i} .
\end{aligned}
$$

We assume the following:

(1) Zero Neumann boundary conditions for the equations

(2) The initial conditions are assumed given

(3) The medium $\Omega$ is vertically homogeneous and take $\Omega \in \mathbf{R}^{2}$

(4) The solutions are smooth and $\Omega$ periodic

(5) $k, \phi, \phi_{1}, d_{2}$, and $K_{i}$ are bounded below by positive constants, and $R_{s}^{\prime}(\widehat{c}), \mu(\widehat{c}), g(\widehat{c}), d_{3}\left(c_{i}\right), f_{i}\left(c_{i}\right)$, and $Q(T)$ are twice continuously differentiable with bounded partial derivatives about the variables in parentheses

(6) $\mathbf{D}=0$

Chou and Li [1], Ewing et al. [2], and Li et al. [3] have presented and studied several numerical methods for system (1)-(5) and its incompressible case. In this paper, we use the mixed finite element method to approximate the fluid problem and treat the brine, heat, and radionuclides by a modified method of characteristic finite element. It is well known that the full discrete approximation scheme is coupled and nonlinear. If simply lagging, the evaluation of the nonlinear items is used to obtain a linear system; it would be inevitable to introduce the constraint conditions about the mesh grid due to the stability requirement. Moreover, it would take an expensive cost to choose the implicit scheme to nonlinear solutions. An efficient method motivated by $\mathrm{Xu}$ [4] is considered in this paper. The method is used by Bi et al. [5], Chen et al. [6-10], and Liu et al. [11, 12] for solving some nonlinear problem. We shall relegate all of nonlinear iterations on a coarse grid of size $H$ much coarser than the original fine grid of size $h$. According to the error estimates in the context, it obtains the asymptotically optimal accuracy to take $H=O\left(h^{1 / 3}\right)$.

The remainder of the paper is organized as follows. Notations and approximation assumptions are given in Section 2. A two-grid method is defined and the convergence error estimates are derived in Section 3. In Section 4, we give some conclusions and extensions.

\section{Notations and Approximation Results}

To analyze the temporal discretization on a time interval $(0, T)$, let $M$ be a positive integer number, $\triangle t=T / M$, $t^{n}=n \triangle t(0 \leq n \leq M)$, and $\varphi^{n}=\varphi\left(\cdot, t^{n}\right)$. Let $L^{p}\left(J ; W^{j, q}(\Omega)\right)$ denote the usual set of functions with the norm

$$
\|\psi\|_{L^{p}\left(J ; W^{j, q}(\Omega)\right)}=\left(\int_{J}\|\psi(\cdot, t)\|_{W^{j, q}(\Omega)}^{p} \mathrm{~d} t\right)^{1 / p},
$$

where if $p=\infty$, the integral is replaced by the essential supremum. Let $l^{p}\left(J ; W^{j, q}(\Omega)\right)$ denote the time discrete analogue to the space $L^{p}\left(J ; W^{j, q}(\Omega)\right)$ with the norm

$$
\|\psi\|_{l^{p}\left(W^{j, q}(\Omega)\right)}=\left(\sum_{n=1}^{N}\left\|\psi^{n}\right\|_{W^{j, q}(\Omega)}^{p} \Delta t\right)^{1 / p} .
$$

Let $W=L^{2}(\Omega)$ and $V=\{v \in H(\operatorname{div} ; \Omega) ; v \cdot \gamma=0\}$. The weak form is presented as follows:

$$
\begin{gathered}
\left(\phi_{1} \frac{\partial p}{\partial t}, w\right)+(\nabla \cdot u, w)=\left(-q+R_{s}^{\prime}(\widehat{c}), w\right), \quad \forall w \in W, \\
(a(\widehat{c}) u, v)-(\nabla \cdot v, p)=0, \\
\left(\phi \frac{\partial \widehat{c}}{\partial t}, z\right)+(u \cdot \nabla \widehat{c}, z)+\left(E_{c} \nabla \widehat{c}, \nabla z\right)=(g(\widehat{c}), z), \\
\left(d_{2} \frac{\partial T}{\partial t}, z\right)+c_{p}(u \cdot \nabla T, z)+\left(\widetilde{E}_{H} \nabla T, \nabla z\right)+\left(d_{1}(p) \frac{\partial p}{\partial t}, z\right)=(Q(u, T, \widehat{c}, p), z), \\
\left(\phi K_{i} \frac{\partial c_{i}}{\partial t}, z\right)+\left(u \cdot \nabla c_{i}, z\right)+\left(E_{c} \nabla c_{i}, \nabla z\right)+\left(d_{3}\left(c_{i}\right) \frac{\partial p}{\partial t}, z\right)=\left(f_{i}\left(\widehat{c}, c_{1}, \ldots, c_{N}\right), z\right),
\end{gathered}
$$

for $z \in H^{1}(\Omega)$ and $i=1, \ldots, N$.

Assume that $V_{h} \times W_{h}$ is the Raviart-Thomas space of index at least $k$ associated with a quasitriangulation of $\Omega$ such that the elements have diameters bounded by $h_{p}$. Let
$M_{h}=M_{h_{c}}$ and $R_{h}=R_{h_{T}}$ be finite-dimensional subspaces of $W^{1, \infty}(\Omega)$ for the approximation of concentrations and temperature, respectively, and we take $M_{h}$ and $R_{h}$ as the piecewise-polynomial space of degree at least $l$ and $r$, 
respectively. As in $[2,11,13]$, the approximation properties for $V_{h} \times W_{h}$ and $M_{h}, R_{h}$ are given by

$$
\begin{gathered}
\inf _{v_{h} \in V_{h}}\left\|v-v_{h}\right\| \leq C\|v\|_{k+1} h_{p}^{k+1}, \\
\inf _{v_{h} \in V_{h}}\left\|\nabla \cdot\left(v-v_{h}\right)\right\| \leq C\left(\|v\|_{k+1}+\|\nabla \cdot v\|_{k+1}\right) h_{p}^{k+1},
\end{gathered}
$$

for $v \in V \cap H^{k+1}(\Omega)^{2}$ and $\nabla \cdot v \in H^{k+1}(\Omega)$, and

$$
\begin{gathered}
\inf _{w_{h} \in W_{h}}\left\|w-w_{h}\right\| \leq C\|w\|_{k+1} h_{p}^{k+1}, \quad w \in H^{k+1}(\Omega), \\
\inf _{z_{h} \in M_{h}}\left\|z-z_{h}\right\|_{1, q} \leq C\|z\|_{l+1, q} h_{c}^{l}, \quad z \in W^{l+1, q}(\Omega), 1 \leq q \leq \infty,
\end{gathered}
$$

$\inf _{z_{h} \in R_{h}}\left\|z-z_{h}\right\|_{1, q} \leq C\|z\|_{r+1, q} h_{T}^{r}, \quad z \in W^{r+1, q}(\Omega), 1 \leq q \leq \infty$.

If the initial solutions $\left\{p_{h}^{0}, u_{h}^{0}, \widehat{c}_{h}^{0}, \check{T}_{h}^{0}, c_{i h}^{0}\right\} \in V_{h} \times$ $W_{h} \times M_{h} \times R_{h} \times M_{h}^{N}$, the characteristics-Galerkin and mixed finite element approximation schemes are to find $\left\{p_{h}^{n}, u_{h}^{n}, \widehat{c}_{h}^{n}, \check{T}_{h}^{n}, c_{i h}^{n}\right\} \in V_{h} \times W_{h} \times M_{h} \times R_{h} \times M_{h}^{N}$ satisfying

$\left(\phi_{1} \frac{p_{h}^{n}-p_{h}^{n-1}}{\Delta t}, w\right)+\left(\nabla \cdot u_{h}^{n}, w\right)=\left(-q+R_{s}^{\prime}\left(\widehat{c}_{h}^{n}\right), w\right), \quad \forall w \in W_{h}$,

$$
\left(a\left(\widehat{c}_{h}^{n}\right) u_{h}^{n}, v\right)-\left(\nabla \cdot v, p_{h}^{n}\right)=0, \quad \forall v \in V_{h}
$$

$\left(\phi \frac{\widehat{c}_{h}^{n}-\widehat{\widehat{c}}_{h}^{n-1}}{\Delta t}, z\right)+\left(E_{c} \nabla \widehat{c}_{h}^{n}, \nabla z\right)=\left(g\left(\widehat{c}_{h}^{n}\right), z\right), \quad \forall z \in M_{h}$,

$$
\begin{aligned}
& \left(d_{2} \frac{\check{T}_{h}^{n}-\check{T}_{h}^{n-1}}{\Delta t}, z\right)+\left(\widetilde{E}_{h} \nabla \check{T}_{h}^{n}, \nabla z\right)+\left(d_{1}\left(p_{h}^{n}\right) \frac{p_{h}^{n}-p_{h}^{n-1}}{\Delta t}, z\right) \\
& =\left(Q\left(u_{h}^{n}, \check{T}_{h}^{n}, \widehat{c}_{h}^{n}, p_{h}^{n}\right), z\right), \quad \forall z \in R_{h},
\end{aligned}
$$

$$
\begin{aligned}
& \left(\phi K_{i} \frac{c_{i h}^{n}-\bar{c}_{i h}^{n-1}}{\Delta t}, z\right)+\left(E_{c} \nabla c_{i h}^{n}, \nabla z\right)+\left(d_{3}\left(c_{i h}^{n}\right) \frac{p_{h}^{n}-p_{h}^{n-1}}{\Delta t}, z\right) \\
& =\left(f_{i}\left(\widehat{c}_{h}^{n}, c_{1 h}^{n}, \ldots, c_{N h}^{n}\right), z\right), \quad \forall z \in M_{h},
\end{aligned}
$$

where $i=1, \ldots, N$ and

$$
\begin{aligned}
& \overline{\widehat{c}}_{h}^{n-1}=\widehat{c}_{h}^{n-1}\left(\bar{x}^{n-1}\right), \\
& \bar{x}^{n-1}=x-\frac{u_{h}^{n} \Delta t}{\phi}, \\
& \bar{c}_{i h}^{n-1}=c_{i h}^{n-1}\left(\bar{x}^{n-1}\right), \\
& \bar{x}^{n-1}=x-\frac{u_{h}^{n} \Delta t}{\left(\phi K_{i}\right)}, \\
& \check{T}_{h}^{n-1}=\check{T}_{h}^{n-1}\left(\bar{x}^{n-1}\right), \\
& \bar{x}^{n-1}=x-\frac{c_{p} u_{h}^{n} \Delta t}{d_{2}} .
\end{aligned}
$$

Remark. If $\bar{x}^{n-1}$ is located outside $\Omega$, we can join $\bar{x}^{n-1}$ with $Y \in \partial \Omega$ so that $\left(\bar{x}^{n-1}-Y\right) /\left\|\bar{x}^{n-1}-Y\right\|$ is the outernormal direction to the boundary $\partial \Omega$ at $Y$. Take $x^{*} \in \Omega$ so that $Y-x^{* n-1}=\bar{x}^{n-1}-Y$, then we define $\bar{x}^{n-1}=x^{* n-1}$.

In order to deduce the error estimates, we employ the elliptic projections by labeling them with tildes.

$$
\begin{aligned}
(\nabla \cdot(u-\widetilde{U}), w)+(p-\widetilde{P}, w)=0, & w \in W_{h}, \\
(a(\widehat{c})(u-\widetilde{U}), v)+(\nabla \cdot v, p-\widetilde{P})=0, & v \in V_{h}, \\
\left(E_{c} \nabla(\widehat{c}-\widetilde{C}), \nabla z\right)+\lambda(\widehat{c}-t \widetilde{C}, z)=0, & z \in M_{h}, \\
\left(E_{H} \nabla(T-\widetilde{T}), \nabla z\right)+\lambda(T-\widetilde{T}, z)=0, & z \in R_{h}, \\
\left(E_{c} \nabla\left(c_{i}-\widetilde{C}_{i}\right), \nabla z\right)+\lambda\left(c_{i}-\widetilde{C}_{i}, z\right)=0, & z \in M_{h},
\end{aligned}
$$

where $\quad\{\widetilde{U}, \widetilde{P}\}: J \longrightarrow V_{h} \times W_{h}, \widetilde{C}: J \longrightarrow M_{h}, \widetilde{T}: J \longrightarrow R_{h}$ and $\widetilde{C}_{i}: J \longrightarrow M_{h}$ for $t \in J$ and introduce the following notations:

$$
\begin{aligned}
\eta & =p-\widetilde{P}, \\
\alpha & =\widetilde{P}-p_{h}, \\
\varrho & =u-\widetilde{U}, \\
\beta & =\widetilde{U}-u_{h}, \\
\widehat{\zeta} & =\widetilde{c}-\widetilde{C}, \\
\widehat{\chi} & =\widetilde{C}-\widehat{c}_{h}, \\
\zeta_{i} & =c_{i}-\widetilde{C}_{i}, \\
\chi_{i} & =\widetilde{C}_{i}-c_{i h}, \\
\theta & =T-\widetilde{T}, \\
\psi & =\widetilde{T}-\check{T}_{h} .
\end{aligned}
$$

Subtracting (19) from (9) and taking $w=d_{t} \alpha^{n-1}$, we get the error equation about the pressure function as follows: 


$$
\begin{aligned}
& \left(\phi_{1} d_{t} \alpha^{n-1}, d_{t} \alpha^{n-1}\right)+\left(\nabla \cdot \beta^{n}, d_{t} \alpha^{n-1}\right) \\
= & -\left(\phi_{1} \frac{\partial \eta^{n}}{\partial t}, d_{t} \alpha^{n-1}\right)+\left(\phi_{1}\left(d_{t} \widetilde{P}^{n-1}-\frac{\partial \widetilde{P}^{n}}{\partial t}\right), d_{t} \alpha^{n-1}\right) \\
& +\left(\frac{\partial R_{s}^{\prime}}{\partial \widehat{c}}\left(\delta_{1}^{n}\right)\left(\hat{c}^{n}-\widehat{c}_{h}^{n}\right), d_{t} \alpha^{n-1}\right),
\end{aligned}
$$

where $d_{t} \alpha^{n-1}=\left(\alpha^{n}-\alpha^{n-1}\right) / \Delta t$ and $\delta_{1}^{n}$ is between $\widehat{c}^{n}$ and $\widehat{c}_{h}^{n}$.

Next, combining (20) from (10) at $t=t^{n}$ with the test function $\beta^{n}$,

$$
\left(a\left(\widehat{c}_{h}^{n}\right) \beta^{n}, \beta^{n}\right)-\left(\nabla \cdot \beta^{n}, \alpha^{n}\right)=-\left(\frac{\partial a}{\partial \widehat{c}}\left(\delta_{2}^{n}\right) \widetilde{U}^{n}\left(\widehat{c}^{n}-\widehat{c}_{h}^{n}\right), \beta^{n}\right) .
$$

When $t=t^{n-1}$, we apply the Taylor expansion and obtain that

$$
\begin{aligned}
& \left(a\left(\widehat{c}_{h}^{n-1}\right) \beta^{n-1}, \beta^{n-1}\right)-\left(\nabla \cdot \beta^{n}, \alpha^{n-1}\right) \\
= & \left(a\left(\widehat{c}_{h}^{n-1}\right) \beta^{n-1}, \beta^{n-1}-\beta^{n}\right)-\left(\frac{\partial a}{\partial \widehat{c}}\left(\delta_{2}^{n-1}\right) \widetilde{U}^{n-1}\left(\widehat{c}^{n-1}-\widehat{c}_{h}^{n-1}\right), \beta^{n}\right),
\end{aligned}
$$

where $\delta_{2}$ is between $\widehat{c}$ and $\widehat{c}_{h}$.

Combining (32) with (33), we get

$$
\begin{aligned}
& \frac{1}{2} d_{t}\left\{\left(a\left(\widehat{c}_{h}^{n-1}\right) \beta^{n-1}, \beta^{n-1}\right)\right\}-\left(\nabla \cdot \beta^{n}, d_{t} \alpha^{n-1}\right) \\
= & -\frac{1}{2 \Delta t}\left(a\left(\widehat{c}_{h}^{n}\right) \beta^{n}, \beta^{n}\right)+\frac{1}{2 \Delta t}\left(a\left(\widehat{c}_{h}^{n-1}\right) \beta^{n}, \beta^{n}\right)-\frac{1}{2 \Delta t}\left(a\left(\widehat{c}_{h}^{n-1}\right) \beta^{n}, \beta^{n}\right) \\
& +\frac{1}{\Delta t}\left(a\left(\widehat{c}_{h}^{n-1}\right) \beta^{n-1}, \beta^{n}\right)-\frac{1}{2 \Delta t}\left(a\left(\widehat{c}_{h}^{n-1}\right) \beta^{n-1}, \beta^{n-1}\right) \\
& -\left(d_{t}\left\{\frac{\partial a}{\partial \widehat{c}}\left(\delta_{2}^{n-1}\right) \widetilde{U}^{n-1}\left(\widehat{c}^{n-1}-\widehat{c}_{h}^{n-1}\right), \beta^{n}\right) .\right.
\end{aligned}
$$

By (31) and (34),

$$
\begin{aligned}
& \left(\phi_{1} d_{t} \alpha^{n-1}, d_{t} \alpha^{n-1}\right)+\frac{1}{2} d_{t}\left\{\left(a\left(\widehat{c}_{h}^{n-1}\right) \beta^{n-1}, \beta^{n-1}\right)\right\} \\
= & -\left(\phi_{1} \frac{\partial \eta^{n}}{\partial t}, d_{t} \alpha^{n-1}\right)+\left(\phi_{1}\left(d_{t} \widetilde{P}^{n-1}-\frac{\partial \widetilde{P}^{n}}{\partial t}\right), d_{t} \alpha^{n-1}\right)+\left(\frac{\partial R_{s}^{\prime}}{\partial \widehat{c}}\left(\delta_{1}^{n}\right)\left(\widehat{c}^{n}-\widehat{c}_{h}^{n}\right), d_{t} \alpha^{n-1}\right) \\
& -\frac{1}{2 \Delta t}\left(\left[a\left(\widehat{c}_{h}^{n}\right)-a\left(\widehat{c}_{h}^{n-1}\right)\right] \beta^{n}, \beta^{n}\right)-\frac{1}{2 \Delta t}\left(a\left(\widehat{c}_{h}^{n-1}\right)\left(\beta^{n}-\beta^{n-1}\right),\left(\beta^{n}-\beta^{n-1}\right)\right) \\
& -\left(d_{t}\left\{\frac{\partial a}{\partial \widehat{c}}\left(\delta_{2}^{n-1}\right) \widetilde{U}^{n-1}\left(\widehat{c}^{n-1}-\widehat{c}_{h}^{n-1}\right), \beta^{n}\right)\right. \\
\leq & -\left(\phi_{1} \frac{\partial \eta^{n}}{\partial t}, d_{t} \alpha^{n-1}\right)+\left(\phi_{1}\left(d_{t} \widetilde{P}^{n-1}-\frac{\partial \widetilde{P}^{n}}{\partial t}\right), d_{t} \alpha^{n-1}\right)+\left(\frac{\partial R_{s}^{\prime}}{\partial \widehat{c}}\left(\delta_{1}^{n}\right)\left(\widehat{c}^{n}-\widehat{c}_{h}^{n}\right), d_{t} \alpha^{n-1}\right) \\
& -\frac{1}{2 \Delta t}\left(\left[a\left(\widehat{c}_{h}^{n}\right)-a\left(\widehat{c}_{h}^{n-1}\right)\right] \beta^{n}, \beta^{n}\right)-\left(d_{t}\left\{\frac{\partial a}{\partial \widehat{c}}\left(\delta_{2}^{n-1}\right) \widetilde{U}^{n-1}\left(\widehat{c}^{n-1}-\widehat{c}_{h}^{n-1}\right), \beta^{n}\right) .\right.
\end{aligned}
$$

Using the deduction as $[1,2]$, we have 


$$
\begin{aligned}
& \left\|d_{t} \alpha^{n-1}\right\|^{2}+\frac{1}{2} d_{t}\left\{\left(a\left(\hat{c}_{h}^{n-1}\right) \beta^{n-1}, \beta^{n-1}\right)\right\} \\
& \leq\left(\left\|\beta^{n}\right\|_{\infty}^{2}+\varepsilon\right)\left\|d_{t} \hat{\chi}^{n-1}\right\|^{2}+C\left(\left\|\hat{\chi}^{n-1}\right\|^{2}+\left\|\beta^{n}\right\|^{2}+h_{p}^{2 k+2}+h_{c}^{2 l+2}+\Delta t^{2}\right) .
\end{aligned}
$$

After making the induction hypothesis that $\sup \left\|\beta^{n}\right\|_{\infty} \longrightarrow 0$, we multiply (36) by $\Delta t$ and sum over $1 \leq n \leq M$ to get

$$
\begin{aligned}
& \sum_{n=1}^{M}\left\|d_{t} \alpha^{n-1}\right\|^{2} \Delta t+\left(a\left(\widehat{c}_{h}^{M-1}\right) \beta^{M}, \beta^{M}\right) \\
& \leq\left(\left\|\beta^{n}\right\|_{\infty}^{2}+\varepsilon\right) \sum_{n=1}^{M}\left\|d_{t} \hat{\chi}^{n-1}\right\|^{2} \Delta t \\
& \quad+C\left(\sum_{n=1}^{M}\left[\left\|\hat{\chi}^{n-1}\right\|_{1}^{2}+\left\|\beta^{n}\right\|^{2}\right] \Delta t+h_{p}^{2 k+2}+h_{c}^{2 l+2}+\Delta t^{2}\right) .
\end{aligned}
$$

Combining (11) and (21), we get the following equality, in which we choose the test function $z=\hat{\chi}^{n}-\hat{\chi}^{n-1}=d_{t} \hat{\chi}^{n-1} \Delta t$ and sum over $1 \leq n \leq M$ :

$$
\begin{aligned}
& \sum_{n=1}^{M}\left(\phi d_{t} \hat{\chi}^{n-1}, d_{t} \hat{\chi}^{n-1}\right) \Delta t+\frac{1}{2}\left(E_{c} \nabla \hat{\chi}^{M}, \nabla \hat{\chi}^{M}\right)-\frac{1}{2}\left(E_{c} \nabla \hat{\chi}^{0}, \nabla \hat{\chi}^{0}\right) \\
& \leq \sum_{n=1}^{M}\left(\phi \frac{\partial \hat{c}^{n}}{\partial t}+u_{h}^{n} \cdot \nabla \hat{\boldsymbol{c}}^{n}-\phi \frac{\hat{c}^{n}-\overline{\bar{c}}^{n-1}}{\Delta t}, d_{t} \hat{\chi}^{n-1}\right) \Delta t+\sum_{n=1}^{M}\left(\phi \frac{\overline{\hat{\chi}}^{n-1}-\hat{\chi}^{n-1}}{\Delta t}, d_{t} \hat{\chi}^{n-1}\right) \Delta t \\
& +\sum_{n=1}^{M}\left(\phi d_{t} \hat{\zeta}^{n-1}, d_{t} \hat{\chi}^{n-1}\right) \Delta t+\sum_{n=1}^{M}\left(\phi \frac{\hat{\zeta}^{n-1}-\bar{\zeta}^{n-1}}{\Delta t}, d_{t} \hat{\chi}^{n-1}\right) \Delta t+\sum_{n=1}^{M} \lambda\left(\hat{\zeta}^{n}, d_{t} \hat{\chi}^{n-1}\right) \Delta t \\
& +\sum_{n=1}^{M}\left(\left(u^{n}-u_{h}^{n}\right) \cdot \nabla \hat{c}^{n}, d_{t} \hat{\chi}^{n-1}\right) \Delta t+\sum_{n=1}^{M}\left(\frac{\partial g}{\partial \widehat{c}}\left(\widehat{c}_{h}^{n}\right)\left(\hat{\zeta}^{n}+\hat{\chi}^{n}\right), d_{t} \hat{\chi}^{n-1}\right) \Delta t
\end{aligned}
$$

where $\quad \overline{\hat{\chi}}^{n-1}=\widehat{\chi}^{n-1}\left(\bar{x}^{n-1}\right), \bar{\zeta}^{n-1}=\widehat{\zeta}^{n-1}\left(\bar{x}^{n-1}\right), \quad$ and $\quad$ Note that $\bar{x}^{n-1}=x-\left(u_{h}^{n} / \phi(x)\right) \Delta t$.

$$
\begin{aligned}
& \left|\sum_{n=1}^{M}\left(\phi \frac{\hat{\zeta}^{n-1}-\overline{\widehat{\zeta}}^{n-1}}{\Delta t}, d_{t} \hat{\chi}^{n-1}\right) \Delta t\right| \\
& =\left|\left[\left(\phi\left(\widehat{\zeta}^{M-1}-\overline{\widehat{\zeta}}^{M-1}\right), \hat{\chi}^{M}\right)-\left(\phi\left(\widehat{\zeta}^{0}-\overline{\widehat{\zeta}}^{0}\right), \hat{\chi}^{0}\right)\right]-\sum_{n=1}^{M-1}\left(\phi d_{t}\left(\hat{\zeta}^{n}-\bar{\zeta}^{n}\right), \hat{\chi}^{n}\right)\right| \\
& =C \Delta t\left\{\left\|\hat{\zeta}^{M-1}\right\|^{2}+\left\|\hat{\zeta}^{0}\right\|^{2}+\sum_{n=1}^{M-1}\left(\left\|\hat{\zeta}^{n-1}\right\|^{2}+\left\|d_{t} \widehat{\zeta}^{n}\right\|^{2}\right)\right\}+\varepsilon \Delta t\left\|\hat{\chi}^{0}\right\|_{1}^{2} .
\end{aligned}
$$

The reminder of the right side items in (38) is just as [2], that is, 


$$
\begin{aligned}
& \sum_{n=1}^{M}\left\|d_{t} \widehat{\chi}^{n-1}\right\|^{2} \Delta t+\left(E_{c} \nabla \hat{\chi}^{M}, \nabla \widehat{\chi}^{M}\right) \\
& \leq C\left(\sum_{n=1}^{M}\left[\left\|\nabla \widehat{\chi}^{n-1}\right\|_{\infty}^{2}\left\|\hat{\chi}^{n-1}\right\|_{1}^{2}+\left\|\beta^{n}\right\|^{2}\right] \Delta t+h_{p}^{2 k+2}+h_{c}^{2 l+2}+\Delta t^{2}\right) .
\end{aligned}
$$

It follows from the assumption sup $\left\|\nabla \widehat{\chi}^{n-1}\right\|_{\infty} \leq C$ and Gronwall lemma that

$$
\begin{aligned}
& \|\widehat{\chi}\|_{L^{\infty}\left(H^{1}\right)}+\left\|d_{t} \hat{\chi}\right\|_{l^{2}\left(L^{2}\right)}+\|\beta\|_{L^{\infty}\left(L^{2}\right)}+\left\|d_{t} \alpha\right\|_{l^{2}\left(L^{2}\right)} \\
& \leq C\left(\Delta t+h_{c}^{l+1}+h_{p}^{k+1}\right) .
\end{aligned}
$$

Then, by the inverse estimate and (40), we know that the induction hypotheses hold if

$$
\begin{aligned}
h_{p}^{k+1} & =o\left(h_{c}\right), \\
h_{c}^{l+1} & =o\left(h_{p}\right), \\
\Delta t & =o\left(h_{c}\right), \\
\Delta t & =o\left(h_{p}\right) .
\end{aligned}
$$

Finally, from the approximation properties,

$$
\begin{aligned}
& \left\|\widehat{c}-\widehat{c}_{h}\right\|_{L^{\infty}\left(h^{1}\right)}+\left\|d_{t}\left(\widehat{c}-\widehat{c}_{h}\right)\right\|_{l^{2}\left(L^{2}\right)}+\left\|u-u_{h}\right\|_{L^{\infty}\left(L^{2}\right)}+\left\|d_{t}\left(p-p_{h}\right)\right\|_{l^{2}\left(L^{2}\right)} \\
& \leq c\left(\Delta t+h_{c}^{l}+h_{p}^{k+1}\right) .
\end{aligned}
$$

Similar to the above analysis, we can obtain the error estimates for the radionuclide equation and heat equation as follows:

$$
\begin{aligned}
\sum_{i=1}^{N}\left\|\chi_{i}\right\|_{L^{\infty}\left(H^{1}\right)}+\sum_{i=1}^{N}\left\|d_{t} \chi_{i}\right\|_{l^{2}\left(L^{2}\right)} \leq C\left(\Delta t+h_{c}^{l+1}+h_{p}^{k+1}\right), \\
\|\psi\|_{L^{\infty}\left(H^{1}\right)}+\left\|d_{t} \psi\right\|_{l^{2}\left(L^{2}\right)} \leq C\left(\Delta t+h_{c}^{l+1}+h_{T}^{r+1}+h_{p}^{k+1}\right),
\end{aligned}
$$

where $\quad h_{p}^{k+1}=o\left(h_{T}\right), h_{c}^{l+1}=o\left(h_{T}\right)$, and $\Delta t=o\left(h_{T}\right) \quad$ are satisfied.

Note that the time step is limited to be $o(h)$ due to the theoretical proof.

Theorem 1. Define $\left\{p_{h}^{n}, u_{h}^{n}, \widehat{c}_{h}^{n}, \check{T}_{h}^{n}, c_{i h}^{n}\right\} \in V_{h} \times W_{h} \times M_{h} \times$ $R_{h} \times M_{h}^{N}$ for $n \geq 1$ by system (19)-(23) and assume that the approximation properties (25)-(29) hold. If

$$
\begin{aligned}
h_{p}^{k+1} & =o\left(h_{c}\right), \\
h_{c}^{l+1} & =o\left(h_{p}\right), \\
\Delta t & =o\left(h_{c}\right), \\
\Delta t & =o\left(h_{p}\right), \\
h_{p}^{k+1} & =o\left(h_{T}\right), \\
h_{c}^{l+1} & =o\left(h_{T}\right), \\
\Delta t & =o\left(h_{T}\right),
\end{aligned}
$$

then there exists a positive constant $C$ independent of $h$ and $\triangle t$, such that

$$
\begin{aligned}
& \left\|\widehat{c}-\widehat{c}_{h}\right\|_{L^{\infty}\left(H^{1}\right)}+\left\|d_{t}\left(\widehat{c}-\widehat{c}_{h}\right)\right\|_{l^{2}\left(L^{2}\right)}+\left\|u-u_{h}\right\|_{L^{\infty}\left(L^{2}\right)}+\left\|d_{t}\left(p-p_{h}\right)\right\|_{l^{2}\left(L^{2}\right)} \\
& \quad+\sum_{i=1}^{N}\left\|c_{i}-c_{i h}\right\|_{L^{\infty}\left(H^{1}\right)}+\sum_{i=1}^{N}\left\|d_{t}\left(c_{i}-c_{i h}\right)\right\|_{l^{2}\left(L^{2}\right)} \\
& \quad+\left\|T-\check{T}_{h}\right\|_{L^{\infty}\left(H^{1}\right)}+\left\|d_{t}\left(T-\breve{T}_{h}\right)\right\|_{l^{2}\left(L^{2}\right)} \\
& \leq C\left(\Delta t+h_{c}^{l}+h_{T}^{r}+h_{p}^{k+1}\right) .
\end{aligned}
$$


Similarly, we can get the error estimates of fine grid scheme in $L^{2}$ norm.

Theorem 2. Define $\left\{p_{h}^{n}, u_{h}^{n}, \widehat{c}_{h}^{n}, \check{T}_{h}^{n}, c_{i h}^{n}\right\} \in V_{h} \times W_{h} \times M_{h} \times$ $R_{h} \times M_{h}^{N}$ for $n \geq 1$ by system (19)-(23) and assume that the approximation properties (25)-(29) hold. Then, there exists a positive constant $C$ independent of $h, H$, and $\triangle t$, such that

$$
\begin{aligned}
& \left\|\widehat{c}-\widehat{c}_{h}\right\|_{L^{\infty}\left(L^{2}\right)}+\left\|u-u_{h}\right\|_{l^{2}\left(L^{2}\right)}+\left\|p-p_{h}\right\|_{L^{\infty}\left(L^{2}\right)} \\
& \quad+\sum_{i=1}^{N}\left\|c_{i}-c_{i h}\right\|_{L^{\infty}\left(L^{2}\right)}+\left\|T-\check{T}_{h}\right\|_{L^{\infty}\left(L^{2}\right)} \\
& \leq C\left(\Delta t+h_{c}^{l+1}+h_{T}^{r+1}+h_{p}^{k+1}\right) .
\end{aligned}
$$

\section{An Efficient Method}

We now use and analyze a two-grid method for iteratively solving the nonlinear problem. The method has two steps.

Stage 1 . On the coarse grid $\mathscr{T}_{H}$ with a mesh size $H \in(0,1)$, solve a small nonlinear system for $\left\{P_{H}^{n}, U_{H}^{n}, \widehat{C}_{H}^{n}, T_{H}^{n}, C_{i H}^{n}\right\} \in V_{H} \times W_{H} \times M_{H} \times R_{H} \times M_{H}^{N}$ given by (19)-(23).

Stage 2. On the fine grid $\mathscr{T}_{h}$ with a mesh size $h \in(0,1)(h \ll H)$, solve the following linear system for $\left\{P_{h}^{n}, U_{h}^{n}, \widehat{C}_{h}^{n}, T_{h}^{n}, C_{i h}^{n}\right\} \in V_{h} \times W_{h} \times M_{h} \times R_{h} \times M_{h}^{N}$ :

$$
\begin{gathered}
\left(\phi_{1} \frac{P_{h}^{n}-P_{h}^{n-1}}{\Delta t}, w\right)+\left(\nabla \cdot U_{h}^{n}, w\right)-\left(\frac{\partial R_{s}^{\prime}}{\partial \widehat{c}}\left(\widehat{C}_{H}^{n}\right) \widehat{C}_{h}^{n}, w\right) \\
=\left(-q+R_{s}^{\prime}\left(\widehat{C}_{H}^{n}\right)-\frac{\partial R_{s}^{\prime}}{\partial \widehat{c}}\left(\widehat{C}_{H}^{n}\right) \widehat{C}_{H}^{n}, w\right), \quad \forall w \in W_{h}, \\
\left(a\left(\widehat{C}_{H}^{n}\right) U_{h}^{n}+\frac{\partial a}{\partial \widehat{c}}\left(\widehat{C}_{H}^{n}\right) U_{H}^{n} \widehat{C}_{h}^{n}, v\right)-\left(\nabla \cdot v, P_{h}^{n}\right)=\left(\frac{\partial a}{\partial \widehat{c}}\left(\widehat{C}_{H}^{n}\right) U_{H}^{n} \widehat{C}_{H}^{n}, v\right), \quad \forall v \in V_{h}, \\
\left(\phi \frac{\widehat{C}_{h}^{n}-\overline{\widehat{C}}_{h}^{n-1}}{\Delta t}, z\right)+\left(U_{h}^{n} \cdot \nabla \widehat{C}_{H}^{n}, z\right)+\left(E_{c} \nabla \widehat{C}_{h}^{n}, \nabla z\right)-\left(\frac{\partial g}{\partial \widehat{c}}\left(\widehat{C}_{H}^{n}\right) \widehat{C}_{h}^{n}, z\right) \\
=\left(U_{H}^{n} \cdot \nabla \widehat{C}_{H}^{n}, z\right)+\left(g\left(\widehat{C}_{H}^{n}\right)-\frac{\partial g}{\partial \widehat{c}}\left(\widehat{C}_{H}^{n}\right) \hat{C}_{H}^{n}, z\right), \quad \forall z \in M_{h}, \\
\left(d_{2} \frac{T_{h}^{n}-\bar{T}_{h}^{n-1}}{\Delta t}, z\right)+\left(\widetilde{E}_{H} \nabla T_{h}^{n}, \nabla z\right)-\left(\frac{\partial Q^{H}}{\partial T} T_{h}^{n}, z\right) \\
=-\left(d_{1}\left(P_{h}^{n}\right) \frac{P_{h}^{n}-P_{h}^{n-1}}{\Delta t}, z\right)+\left(Q^{H}, z\right)-\left(\frac{\partial Q^{H}}{\partial T} T_{H}^{n}, z\right), \quad \forall z \in R_{h}, \\
+\left(\frac{\partial d_{3}}{\partial c_{i}}\left(C_{i H}^{n}\right) \frac{P_{h}^{n}-P_{h}^{n-1}}{\Delta t} C_{i H}^{n}+f_{i}^{H}-\frac{\partial f_{i}^{H}}{\partial c_{1}} C_{1 H}^{n}-\cdots-\frac{\partial f_{i}^{H}}{\partial c_{N}} C_{N H}^{n}, z\right), \quad \forall z \in M_{h}, \\
\left.\phi K_{i} \frac{C_{i h}^{n}-\bar{C}_{i h}^{n-1}}{\Delta t}, z\right)+\left(E_{c} \nabla C_{i H}^{n}, \nabla z\right)+\left(\frac{\partial d_{3}}{\partial c_{i}}\left(C_{i H}^{n}\right) \frac{P_{h}^{n}-P_{h}^{n-1}}{\Delta t} C_{i h}^{n}, z\right) \\
-\left(\frac{\partial f_{i}^{H}}{\partial c_{1}} C_{1 h}^{n}+\cdots+\frac{\partial f_{i}^{H}}{\partial c_{N}} C_{N h}^{n}, z\right)=-\left(d_{3}\left(C_{i H}^{n}\right) \frac{P_{h}^{n}-P_{h}^{n-1}}{\Delta t}, z\right)
\end{gathered}
$$

where 


$$
\begin{aligned}
Q^{H} & =Q\left(U_{h}^{n}, T_{H}^{n}, \widehat{C}_{h}^{n}, P_{h}^{n}\right), \\
f_{i}^{H} & =f_{i}\left(\widehat{C}_{h}^{n}, C_{1 H}^{n}, \ldots, C_{N H}^{n}\right), \\
\widehat{C}_{h}^{n-1} & =\widehat{C}_{h}^{n-1}\left(\bar{x}^{n-1}\right), \\
\bar{x}^{n-1} & =x-\left(\frac{U_{H}^{n} \Delta t}{\phi}\right), \\
\bar{C}_{i h}^{n-1} & =C_{i h}^{n-1}\left(\bar{x}^{n-1}\right), \\
\bar{x}^{n-1} & =x-\left(\frac{U_{h}^{n} \Delta t}{\left(\phi K_{i}\right)}\right), \\
\bar{T}_{h}^{n-1} & =T_{h}^{n-1}\left(\bar{x}^{n-1}\right), \\
\bar{x}^{n-1} & =x-\left(\frac{c_{p} U_{h}^{n} \Delta t}{d_{2}}\right),
\end{aligned}
$$

and the projection on the fine grid is based on the numerical solutions on coarse grid.

The sequential solution processes are defined as follows. Firstly, we apply the Newton iteration to the coupled system on the coarse grid and obtain $\left\{P_{H}^{n}, U_{H}^{n}, \widehat{C}_{H}^{n}, T_{H}^{n}, C_{i H}^{n}\right\}$. Next, combining (48)-(50), we get $P_{h}^{n}$ and $U_{h}^{n}$ with $R T_{1}$ and $\widehat{C}_{h}^{n}$ with piecewise linear finite element using a coupled linear system. Finally, from (51) and (52), we can get $C_{i h}^{n}$ and $T_{h}^{n}$ by parallel computation.

In order to analyze the linear scheme on the fine grid, we define

$$
\begin{aligned}
\pi & =\widetilde{P}-P_{h}, \\
\sigma & =\widetilde{U}-U_{h}, \\
\widehat{\xi} & =\widetilde{C}-\widehat{C}_{h}, \\
\xi & =\widetilde{C}_{i}-C_{i h}, \\
\omega & =\widetilde{T}-T_{h} .
\end{aligned}
$$

According to Taylor expansion, there exists a positive $\delta_{3}$ such that

$$
\begin{aligned}
R_{s}^{\prime}\left(\widehat{c}^{n}\right)-R_{s}^{\prime}\left(\widehat{C}_{H}^{n}\right)-\frac{\partial R_{s}^{\prime}}{\partial \widehat{c}}\left(\widehat{C}_{H}^{n}\right)\left(\widehat{C}_{h}^{n}-\widehat{C}_{H}^{n}\right) \\
=R_{s}^{\prime}\left(\widehat{C}_{H}^{n}\right)+\frac{\partial R_{s}^{\prime}}{\partial \widehat{c}}\left(\widehat{C}_{H}^{n}\right)\left(\widehat{c}^{n}-\widehat{C}_{H}^{n}\right)-R_{s}^{\prime}\left(\widehat{C}_{H}^{n}\right)-\frac{\partial R_{s}^{\prime}}{\partial \widehat{c}}\left(\widehat{C}_{H}^{n}\right)\left(\widehat{C}_{h}^{n}-\widehat{C}_{H}^{n}\right) \\
\quad+\frac{\partial^{2} R_{s}^{\prime}}{2 \partial \widehat{c}^{2}}\left(\delta_{3}\right)\left(\widehat{c}^{n}-\widehat{C}_{H}^{n}\right)^{2} \\
=\frac{\partial R_{s}^{\prime}}{\partial \widehat{c}}\left(\widehat{C}_{H}^{n}\right)\left(\widehat{c}^{n}-\widehat{C}_{h}^{n}\right)+\frac{\partial^{2} R_{s}^{\prime}}{2 \partial \widehat{c}^{2}}\left(\delta_{3}\right)\left(\widehat{c}^{n}-\widehat{C}_{H}^{n}\right)^{2} .
\end{aligned}
$$

According to (48), (9), and (25),

$$
\begin{aligned}
\left(\phi_{1} d_{t} \pi^{n-1}, d_{t} \pi^{n-1}\right)+\left(\nabla \cdot \sigma^{n}, d_{t} \pi^{n-1}\right) \\
=-\left(\phi_{1} \frac{\partial \eta^{n}}{\partial t}, d_{t} \pi^{n-1}\right)+\left(\phi_{1}\left(d_{t} \widetilde{P}^{n-1}-\frac{\partial \widetilde{P}^{n}}{\partial t}\right), d_{t} \pi^{n-1}\right)+\left(\eta^{n}, d_{t} \pi^{n-1}\right) \\
\quad+\left(\frac{\partial R_{s}^{\prime}}{\partial \widehat{c}}\left(\widehat{C}_{H}^{n}\right)\left(\widehat{c}^{n}-\widehat{C}_{h}^{n}\right)+\frac{\partial^{2} R_{s}^{\prime}}{2 \widehat{\widehat{c}}^{2}}\left(\delta_{1}\right)\left(\widehat{c}^{n}-\widehat{C}_{H}^{n}\right)^{2}, d_{t} \pi^{n-1}\right)
\end{aligned}
$$

Like the deduction of (34), we see that 


$$
\begin{aligned}
& \frac{1}{2} d_{t}\left\{\left(a\left(\widehat{C}_{H}^{n-1}\right) \sigma^{n-1}, \sigma^{n-1}\right)\right\}-\left(\nabla \cdot \sigma^{n}, d_{t} \pi^{n-1}\right) \\
& =-\frac{1}{2 \Delta t}\left(a\left(\widehat{C}_{H}^{n}\right) \sigma^{n}, \sigma^{n}\right)+\frac{1}{2 \Delta t}\left(a\left(\widehat{C}_{H}^{n-1}\right) \sigma^{n}, \sigma^{n}\right)-\frac{1}{2 \Delta t}\left(a\left(\widehat{C}_{H}^{n-1}\right) \sigma^{n}, \sigma^{n}\right) \\
& \quad+\frac{1}{\Delta t}\left(a\left(\widehat{C}_{H}^{n-1}\right) \sigma^{n-1}, \sigma^{n}\right)-\frac{1}{2 \Delta t}\left(a\left(\widehat{C}_{H}^{n-1}\right) \sigma^{n-1}, \sigma^{n-1}\right)-\left(d _ { t } \left\{\frac{\partial a}{\partial \widehat{c}}\left(\widehat{C}_{H}^{n-1}\right) \widetilde{U}^{n-1}\right.\right. \\
& \left.\left.\left(\widehat{c}^{n-1}-\widehat{C}_{h}^{n-1}\right)+\frac{\partial a}{\partial \widehat{c}}\left(\widehat{C}_{H}^{n-1}\right)\left(\widetilde{U}^{n-1}-U_{H}^{n-1}\right)\left(\widehat{C}_{h}^{n-1}-\widehat{C}_{H}^{n-1}\right)\right\}, \sigma^{n}\right) \\
& \quad-\frac{1}{2 \Delta t}\left(\frac{\partial^{2} a}{\partial \widehat{c}^{2}}\left(\delta_{4}\right) \widetilde{U}^{n}\left(\widehat{c}^{n}-\widehat{C}_{H}^{n}\right)^{2}-\frac{\partial^{2} a}{\partial \widehat{c}^{2}}\left(\delta_{4}\right) \widetilde{U}^{n-1}\left(\widehat{c}^{n-1}-\widehat{C}_{H}^{n-1}\right)^{2}, \sigma^{n}\right),
\end{aligned}
$$

where $\delta_{4}$ is between $\widehat{c}$ and $\widehat{C}_{H}$.

Hence, (56) and (57) can give that

$$
\begin{aligned}
& \left(\phi_{1} d_{t} \pi^{n-1}, d_{t} \pi^{n-1}\right)+\frac{1}{2} d_{t}\left\{\left(a\left(\widehat{C}_{H}^{n-1}\right) \sigma^{n-1}, \sigma^{n-1}\right)\right\} \\
= & -\left(\phi_{1} \frac{\partial \eta^{n}}{\partial t}, d_{t} \pi^{n-1}\right)+\left(\phi_{1}\left(d_{t} \widetilde{P}^{n-1}-\frac{\partial \widetilde{P}^{n}}{\partial t}\right), d_{t} \pi^{n-1}\right)+\left(\eta^{n}, d_{t} \pi^{n-1}\right) \\
& +\left(\frac{\partial R_{s}^{\prime}}{\partial \widehat{c}}\left(\widehat{C}_{H}^{n}\right)\left(\widehat{c}^{n}-\widehat{C}_{h}^{n}\right)+\frac{\partial^{2} R_{s}^{\prime}}{2 \widehat{c}^{2}}\left(\delta_{3}\right)\left(\widehat{c}^{n}-\widehat{C}_{H}^{n}\right)^{2}, d_{t} \pi^{n-1}\right) \\
& -\frac{1}{2 \Delta t}\left(\left[a\left(\widehat{C}_{H}^{n}\right)-a\left(\widehat{C}_{H}^{n-1}\right)\right] \sigma^{n}, \sigma^{n}\right)-\frac{1}{2 \Delta t}\left(a\left(\widehat{C}_{H}^{n-1}\right)\left(\sigma^{n}-\sigma^{n-1}\right),\left(\sigma^{n}-\sigma^{n-1}\right)\right), \\
& -\left(d_{t}\left\{\frac{\partial a}{\partial \widehat{c}}\left(\widehat{C}_{H}^{n-1}\right) \widetilde{U}^{n-1}\left(\widehat{c}^{n-1}-\widehat{C}_{h}^{n-1}\right)+\frac{\partial a}{\partial \widehat{c}}\left(\widehat{C}_{H}^{n-1}\right)\left(\widetilde{U}^{n-1}-U_{H}^{n-1}\right)\left(\widehat{C}_{h}^{n-1}-\widehat{C}_{H}^{n-1}\right)\right\}, \sigma^{n}\right) \\
& -\frac{1}{2 \Delta t}\left(\frac{\partial^{2} a}{\partial \widehat{c}^{2}}\left(\delta_{4}\right) \widetilde{U}^{n}\left(\widehat{c}^{n}-\widehat{C}_{H}^{n}\right)^{2}-\frac{\partial^{2} a}{\partial \widehat{c}^{2}}\left(\delta_{4}\right) \widetilde{U}^{n-1}\left(\widehat{c}^{n-1}-\widehat{C}_{H}^{n-1}\right)^{2}, \sigma^{n}\right) .
\end{aligned}
$$

The error equation about $\widehat{c}$ shows that

$$
\begin{aligned}
& \left(\phi \frac{\widehat{\xi}^{n}-\widehat{\xi}^{n-1}}{\Delta t}, z\right)+\left(E_{c} \nabla \widehat{\xi}^{n}, \nabla z\right) \\
& =-\left(\phi \frac{\partial \widehat{c}^{n}}{\partial t}+u_{H}^{n} \cdot \nabla \widehat{c}^{n}-\phi \frac{\widehat{c}^{n}-\overline{\widehat{c}}^{n-1}}{\Delta t}, z\right)+\left(\phi \frac{\bar{\xi}^{n-1}-\widehat{\xi}^{n-1}}{\Delta t}, z\right) \\
& -\left(\phi \frac{\widehat{\zeta}^{n}-\bar{\zeta}^{n-1}}{\Delta t}, z\right)+\lambda\left(\widehat{\zeta}^{n}, z\right)-\left(\left(u^{n}-U_{H}^{n}\right) \cdot\left(\nabla \widehat{c}^{n}-\nabla \widehat{C}_{H}^{n}\right)+\left(u^{n}-U_{h}^{n}\right) \cdot \nabla \widehat{C}_{H}^{n}, z\right) \\
& +\left(\frac{\partial g}{\partial \widehat{c}}\left(\widehat{C}_{H}^{n}\right)\left(\widehat{\zeta}^{n}+\widehat{\xi}^{n}\right), z\right)+\left(\frac{\partial^{2} g}{2 \partial \widehat{c}^{2}}\left(\delta_{5}\right)\left(\widehat{c}^{n}-\widehat{C}_{H}^{n}\right)^{2}, z\right),
\end{aligned}
$$


where $\overline{\bar{\xi}}^{n-1}=\widehat{\xi}^{n-1}\left(\bar{X}^{n-1}\right), \overline{\bar{\zeta}}^{n-1}=\widehat{\zeta}^{n-1}\left(\bar{X}^{n-1}\right)$, and $\bar{X}^{n-1}=$ $x-\left(U_{H}^{n} / \phi(x)\right) \Delta t$.
Taking the test function $z=\widehat{\xi}^{n}-\widehat{\xi}^{n-1}=d_{t} \widehat{\xi}^{n-1} \Delta t$ and summing over $1 \leq n \leq M$, we have

$$
\begin{aligned}
& \sum_{n=1}^{M}\left(\phi d_{t} \widehat{\xi}^{n-1}, d_{t} \widehat{\xi}^{n-1}\right) \Delta t+\frac{1}{2}\left(E_{c} \nabla \widehat{\xi}^{M}, \nabla \widehat{\xi}^{M}\right)-\frac{1}{2}\left(E_{c} \nabla \widehat{\xi}^{0}, \nabla \widehat{\xi}^{0}\right) \\
& \leq \sum_{n=1}^{M}\left(\phi \frac{\partial \widehat{c}^{n}}{\partial t}+u_{H}^{n} \cdot \nabla \widehat{c}^{n}-\phi \frac{\widehat{c}^{n}-\overline{\hat{c}}^{n-1}}{\Delta t}, d_{t} \widehat{\xi}^{n-1}\right) \Delta t+\sum_{n=1}^{M}\left(\phi \frac{\overline{\hat{\xi}}^{n-1}-\hat{\xi}^{n-1}}{\Delta t}, d_{t} \widehat{\xi}^{n-1}\right) \Delta t \\
& +\sum_{n=1}^{M}\left(\phi \frac{\hat{\zeta}^{n}-\overline{\bar{\zeta}}^{n-1}}{\Delta t}, d_{t} \hat{\xi}^{n-1}\right) \Delta t+\sum_{n=1}^{M} \lambda\left(\hat{\zeta}^{n}, d_{t} \hat{\xi}^{n-1}\right) \Delta t \\
& +\sum_{n=1}^{M}\left(\left(u^{n}-U_{H}^{n}\right) \cdot\left(\nabla \widehat{c}^{n}-\nabla \widehat{C}_{H}^{n}\right)+\left(u^{n}-U_{h}^{n}\right) \cdot \nabla \widehat{C}_{H}^{n}, d_{t} \widehat{\xi}^{n-1}\right) \Delta t \\
& +\sum_{n=1}^{M}\left(\frac{\partial g}{\partial \widehat{c}}\left(\widehat{C}_{H}^{n}\right)\left(\hat{\zeta}^{n}+\widehat{\xi}^{n}\right)+\frac{\partial^{2} g}{2 \partial \widehat{c}^{2}}\left(\delta_{4}\right)\left(\hat{c}^{n}-\widehat{C}_{H}^{n}\right)^{2}, d_{t} \hat{\xi}^{n-1}\right) \Delta t .
\end{aligned}
$$

Since

$$
\begin{aligned}
\left\|\left(\widehat{c}-\widehat{C}_{H}\right)^{2}\right\| & \leq\left\|\widehat{c}-\widehat{C}_{H}\right\|_{L^{\infty}}\left\|\widehat{c}-\widehat{C}_{H}\right\|_{L^{2}} \\
& \leq\left(\|\widehat{c}-\widetilde{C}\|_{L^{\infty}}+\left\|\widetilde{C}-\widehat{C}_{H}\right\|_{L^{\infty}}\right)\left\|\left(\widehat{c}-\widehat{C}_{H}\right)\right\|_{L^{2}} \\
& \leq C\left(\left|\ln H_{c}\right| H_{c}^{l+1}+\left|\ln H_{c}\right|\left(H_{c}^{l+1}+H_{p}^{k+1}\right)\right) \\
& \times\left(H_{c}^{l+1}+H_{p}^{k+1}\right) \\
& \leq C\left(H_{c}^{2 l+2}\left|\ln H_{c}\right|+H_{p}^{2 k+2}\left|\ln H_{c}\right|\right),
\end{aligned}
$$$$
\left\|\left(u-U_{H}\right)\left(\widehat{c}-\widehat{C}_{H}\right)\right\| \leq\left\|u-U_{H}\right\|\left\|\widehat{c}-\widehat{C}_{H}\right\|_{L^{\infty}}
$$$$
\left.\leq C\left(H_{c}^{l+1}+H_{p}^{k+1}\right)\right)
$$$$
\times\left(\left|\ln H_{c}\right|\left(H_{c}^{l+1}+H_{p}^{k+1}\right)\right)
$$$$
\leq C\left(H_{c}^{2 l+2}\left|\ln H_{c}\right|+H_{p}^{2 k+2}\left|\ln H_{c}\right|\right) \text {, }
$$$$
\left\|\left(u-U_{H}\right)\left(\nabla \widehat{c}-\nabla \widehat{C}_{H}\right)\right\| \leq\left\|u-U_{H}\right\|\left\|\widehat{c}-\widehat{C}_{H}\right\|_{1, \infty}
$$$$
\leq C\left(H_{c}^{l+1}+H_{p}^{k+1}\right)
$$$$
\times\left(H_{c}^{l}+H_{c}^{-1}\left(H_{c}^{l+1}+H_{p}^{k+1}\right)\right)
$$$$
\leq C\left(H_{c}^{2 l+1}+H_{p}^{2 k+2} H_{c}^{-1}\right) \text {. }
$$

It follows from (61)-(63) that

$$
\begin{aligned}
\| \widehat{c} & -\widehat{C}_{h}\left\|_{L^{\infty}\left(H^{1}\right)}+\right\| d_{t}\left(\widehat{c}-\widehat{C}_{h}\right)\left\|_{L^{2}\left(L^{2}\right)}+\right\| u-U_{H} \|_{L^{\infty}\left(L^{2}\right)} \\
& +\left\|d_{t}\left(p-P_{h}\right)\right\|_{l^{2}\left(L^{2}\right)} \\
\leq & C\left(\Delta t+h_{c}^{l}+h_{p}^{k+1}+H_{c}^{2 l+1}+H_{p}^{2 k+2} H_{c}^{-1}\right) .
\end{aligned}
$$

At last, we present the error results of the radionuclide equation and heat equation as follows:

$$
\begin{aligned}
& \sum_{i=1}^{N}\left\|c_{i}-C_{i h}\right\|_{L^{\infty}\left(H^{1}\right)}+\sum_{i=1}^{N}\left\|d_{t}\left(c_{i}-C_{i h}\right)\right\|_{l^{2}\left(L^{2}\right)} \\
& \leq C\left(\Delta t+h_{c}^{l}+h_{p}^{k+1}+H_{c}^{2 l+2}\left|\ln H_{c}\right|+H_{p}^{2 k+2}\left|\ln H_{c}\right|\right), \\
& \left\|T-T_{h}\right\|_{L^{\infty}\left(H^{1}\right)}+\left\|d_{t}\left(T-T_{h}\right)\right\|_{l^{2}\left(L^{2}\right)} \\
& \leq C\left(\Delta t+h_{c}^{l}+h_{T}^{r}+h_{p}^{k+1}+H_{T}^{2 r+2}\left|\ln H_{T}\right|+H_{c}^{2 l+2}|\ln H|_{T}+H_{p}^{2 k+2}\left|\ln H_{T}\right|\right) .
\end{aligned}
$$


If setting $l=1, r=1, k=1, \quad h_{c}=h_{T}=h_{p}, \quad$ and $H_{c}=H_{T}=H_{p}$, which accords with the assumption (45), we can get the following theorem of fine grid scheme.
Theorem 3. Define $\left\{P_{h}^{n}, U_{h}^{n}, \widehat{C}_{h}^{n}, T_{h}^{n}, C_{i h}^{n}\right\} \in V_{h} \times W_{h} \times M_{h} \times$ $R_{h} \times M_{h}^{N}$ for $n \geq 1$ by system (48)-(52) and assume that the approximation properties (14)-(18) hold. Then, there exists a positive constant $C$ independent of $h, H$, and $\triangle t$, such that

$$
\begin{aligned}
& \left\|\widehat{c}-\widehat{C}_{h}\right\|_{L^{\infty}\left(H^{1}\right)}+\left\|d_{t}\left(\widehat{c}-\widehat{C}_{h}\right)\right\|_{l^{2}\left(L^{2}\right)}+\left\|u-U_{h}\right\|_{L^{\infty}\left(L^{2}\right)}+\left\|d_{t}\left(p-P_{h}\right)\right\|_{l^{2}\left(L^{2}\right)} \\
& \quad+\sum_{i=1}^{N}\left\|c_{i}-C_{i h}\right\|_{L^{\infty}\left(H^{1}\right)}+\sum_{i=1}^{N}\left\|d_{t}\left(c_{i}-C_{i h}\right)\right\|_{l^{2}\left(L^{2}\right)} \\
& \quad+\left\|T-T_{h}\right\|_{L^{\infty}\left(H^{1}\right)}+\left\|d_{t}\left(T-T_{h}\right)\right\|_{l^{2}\left(L^{2}\right)} \\
& \leq C\left(\Delta t+h+H^{3}\right) .
\end{aligned}
$$

Theorem 4. Define $\left\{P_{h}^{n}, U_{h}^{n}, \widehat{C}_{h}^{n}, T_{h}^{n}, C_{i h}^{n}\right\} \in V_{h} \times W_{h} \times M_{h} \times$ $R_{h} \times M_{h}^{N}$ for $n \geq 1$ by system (48)-(52) and assume that the approximation properties (14)-(18) hold. Then, there exists a positive constant $C$ independent of $h, H$, and $\triangle t$, such that

$$
\begin{aligned}
& \left\|\widehat{c}-\widehat{C}_{h}\right\|_{L^{\infty}\left(L^{2}\right)}+\left\|u-U_{h}\right\|_{L^{2}\left(L^{2}\right)}+\left\|p-P_{h}\right\|_{L^{\infty}\left(L^{2}\right)} \\
& \quad+\sum_{i=1}^{N}\left\|c_{i}-C_{i h}\right\|_{L^{\infty}\left(L^{2}\right)}+\left\|T-T_{h}\right\|_{L^{\infty}\left(L^{2}\right)} \\
& \leq C\left(\Delta t+h^{2}+H^{3}\right) .
\end{aligned}
$$

\section{Conclusions and Extensions}

The two-grid method presented in this paper reduces the complexity of problem. It involves a small nonlinear system on a coarse grid of size $H$ and a linear system on a fine grid of size $h$. It is shown that the coarse space can be extremely coarse and still achieve asymptotically optimal approximation as long as the mesh sizes satisfy $H=O\left(h^{1 / 3}\right)$ in $H^{1}$ norm. Compared with the implicit scheme, the two-grid method reduces CPU time. Moreover, the method is suitable to make the large-scale computation and long time duration. The future work is to use the discontinuous finite volume element method [14], block-centered finite difference method [15], higher-order finite volume [16], and SAV method $[17,18]$ to consider this problem.

\section{Data Availability}

No data were used to support this study.

\section{Conflicts of Interest}

The author declares that there are no conflicts of interest.

\section{Acknowledgments}

The author would like to thank Professor Luping Wang whose comments helped improve this paper.

\section{References}

[1] S.-H. Chou and Q. Li, "Characteristics-Galerkin and mixed finite element approximation of contamination by compressible nuclear waste-disposal in porous media," Numerical Methods for Partial Differential Equations, vol. 12, no. 3, pp. 315-332, 1996.

[2] R. E. Ewing, Y. Yuan, and G. Li, "Timestepping along characteristics for a mixed finite-element approximation for compressible flow of contamination from nuclear waste in porous media," SIAM Journal on Numerical Analysis, vol. 26, no. 6, pp. 1513-1524, 1989.

[3] C. Li, Y. Yuan, and H. Song, "An upwind approximation combined with mixed volume element for a positive semidefinite contamination treatment from nuclear waste," Engineering With Computers, vol. 36, no. 4, pp. 1599-1614, 2019.

[4] J. Xu, "Two-grid discretization techniques for linear and nonlinear PDEs," SIAM Journal on Numerical Analysis, vol. 33, no. 5, pp. 1759-1777, 1996.

[5] C. Bi, Y. Lin, and M. Yang, "Finite volume element method for monotone nonlinear elliptic problems," Numerical Methods for Partial Differential Equations, vol. 29, no. 4, pp. 1097-1120, 2013.

[6] C. Chen, H. Liu, X. Zheng, and H. Wang, "A two-grid MMOC finite element method for nonlinear variable-order timefractional mobile/immobile advection-diffusion equations," Computers \& Mathematics with Applications, vol. 79, no. 9, pp. 2771-2783, 2020.

[7] C. Chen, X. Zhang, G. Zhang, and Y. Zhang, "A two-grid finite element method for nonlinear parabolic integro-differential equations," International Journal of Computer Mathematics, vol. 96, no. 10, pp. 2010-2023, 2019.

[8] C. Chen, W. Liu, and C. Bi, "A two-grid characteristic finite volume element method for semilinear advection-dominated diffusion equations," Numerical Methods for Partial Differential Equations, vol. 29, no. 5, pp. 1543-1562, 2013.

[9] C. Chen and X. Zhao, "A posteriori error estimate for finite volume element method of the parabolic equations," $\mathrm{Nu}$ merical Methods for Partial Differential Equations, vol. 33, no. 1, pp. 259-275, 2017.

[10] C. Chen, X. Zhao, and Y. Zhang, "A posteriori error estimate for finite volume element method of the second-order hyperbolic equations," Mathematical Problems in Engineering, vol. 2015, Article ID 510241, 11 pages, 2015.

[11] W. Liu, X. Li, and Q. Zhao, "A two-grid expanded mixed element method for nonlinear non-fickian flow model in 
porous media," International Journal of Computer Mathematics, vol. 91, no. 6, pp. 1299-1314, 2014.

[12] W. Liu, "A two-grid method for the semi-linear reactiondiffusion system of the solutes in the groundwater flow by finite volume element," Mathematics and Computers in Simulation, vol. 142, pp. 34-50, 2017.

[13] F. A. Milner, "Mixed finite element methods for quasilinear second-order elliptic problems," Mathematics of Computation, vol. 44, no. 170, p. 303, 1985.

[14] C. Bi and M. Liu, "A discontinuous finite volume element method for second-order elliptic problems," Numerical Methods for Partial Differential Equations, vol. 28, no. 2, pp. 425-440, 2012.

[15] W. Liu, J. Cui, and J. Xin, "A block-centered finite difference method for an unsteady asymptotic coupled model in fractured media aquifer system," Journal of Computational and Applied Mathematics, vol. 337, pp. 319-340, 2018.

[16] M. Yang, "Higher-order finite volume element methods based on Barlow points for one-dimensional elliptic and parabolic problems," Numerical Methods for Partial Differential Equations, vol. 31, no. 4, pp. 977-994, 2015.

[17] Z. Liu and X. Li, "The exponential scalar auxiliary variable (ESAV) approach for phase field models and its explicit computing," SIAM Journal on Scientific Computing, vol. 42, no. 3, pp. B630-B655, 2020.

[18] Z. Liu and X. Li, "Efficient modified techniques of invariant energy quadratization approach for gradient flows," Applied Mathematics Letters, vol. 98, pp. 206-214, 2019. 